\title{
Comparison of Clinical Features between the High and Low Serum KL-6 Patients with Acute Exacerbation of Interstitial Lung Diseases
}

\author{
Yoichi Tagami ${ }^{1}$, ${ }^{1,2,3,4}$ Yu Hara ${ }^{(D)},{ }^{1,2,3,4}$ Kota Murohashi, ${ }^{1,2,3,4}$ Ryo Nagasawa, ${ }^{1,2,3,4}$ \\ Yurika Nishikawa, ${ }^{1,2,3,4}$ Meiro Tanaka, ${ }^{1,2,3,4}$ Ayako Aoki, ${ }^{1,2,3,4}$ Katsushi Tanaka, ${ }^{1,2,3,4}$ \\ Kentaro Nakashima, ${ }^{1,2,3,4}$ Keisuke Watanabe, ${ }^{1,2,3,4}$ Nobuyuki Horita, ${ }^{1,2,3,4}$ \\ Nobuaki Kobayashi $\mathbb{D}^{1,2,3,4}$ Masaki Yamamoto, ${ }^{1,2,3,4}$ Makoto Kudo, ${ }^{1,2,3,4}$ Koji Okudela, , ,2,3,4 \\ and Takeshi Kaneko ${ }^{1,2,3,4}$ \\ ${ }^{1}$ Department of Pulmonology, Yokohama City University Graduate School of Medicine, Yokohama, Japan \\ ${ }^{2}$ Department of Respiratory Medicine, Kanagawa Cardiovascular and Respiratory Center, Yokohama, Japan \\ ${ }^{3}$ Respiratory Disease Center, Yokohama City University Medical Center, Yokohama, Japan \\ ${ }^{4}$ Department of Pathology, Yokohama City University Graduate School of Medicine, Yokohama, Japan \\ Correspondence should be addressed to Yu Hara; bronchiole0723@yahoo.co.jp
}

Received 10 June 2021; Revised 12 October 2021; Accepted 15 November 2021; Published 30 November 2021

Academic Editor: Akiteru Goto

Copyright (C) 2021 Yoichi Tagami et al. This is an open access article distributed under the Creative Commons Attribution License, which permits unrestricted use, distribution, and reproduction in any medium, provided the original work is properly cited.

Background. Serum Krebs von den Lungen-6 (KL-6) measurement is widely used to assess disease activity or prognosis in patients with interstitial lung diseases (ILDs). However, the clinical differences between high and low serum KL-6 levels at the time of acute exacerbation (AE) of ILD are not well known. Methods. Clinical parameters including age, sex, Charlson Comorbidity Index score (CCIS), blood biomarkers, high-resolution CT findings, and disease mortality were retrospectively compared between high and low KL-6 (cutoff value: $1000 \mathrm{U} / \mathrm{mL}$ ) patients at the time of diagnosis of AE of ILDs. Results. Thirty-eight high serum KL-6 and 57 low serum KL-6 patients were included. There was no significant difference in 6-month mortality between them $(P=0.685)$, whereas serum lactate dehydrogenase was a significant predictor of 6-month mortality in the high serum KL-6 patients (odds ratio (OR): 1.006; 95\% confidence interval (CI): 1.003-1.009; $P<0.001$ ), and CCIS (OR: 1.502; 95\% CI: $1.242-1.838 ; P<0.001$ ) and sex (OR: $5.751 ; 95 \%$ CI: $1.121-105.163 ; P=0.033$ ) were significant predictors in low serum KL-6 patients. In addition, the incidences of congestive heart failure, symptomatic chronic pulmonary disease, cerebrovascular disease, and second metastatic solid tumours were significantly higher in nonsurvivors with low serum KL-6 than in other groups $(P<0.05)$. Conclusions. The clinical features in patients with AEs of ILDs may differ depending on the serum KL-6 level, and clinicopathological examination according to this subtyping guided by the serum KL-6 level is essential.

\section{Introduction}

The prognosis of acute exacerbations (AEs) of interstitial lung diseases (ILDs) such as idiopathic interstitial pneumonias (IIPs), chronic hypersensitivity pneumonitis, and connective tissue disease-associated ILDs (CTD-ILDs) is generally poor $[1,2]$. Furthermore, the pathological findings of patients with AEs of ILDs show not only diffuse alveolar damage (DAD) but also a variety of pathological conditions including organizing pneumonia (OP), diffuse alveolar haemorrhage (DAH), lung cancer, and bronchopneumonia [3]. Despite pathological heterogeneity, it is very difficult to perform a lung biopsy during an $\mathrm{AE}$ due to severe respiratory failure. Therefore, it is necessary to plan the treatment strategies or attempt to accurately predict the disease prognosis using less invasive modalities such as symptoms, blood test results, and imaging findings in the clinical setting. 
Krebs von den Lungen-6 (KL-6) is a high-molecularweight mucin-like glycoprotein, also known as human mucin-1 (MUC1). It is expressed mainly on bronchiolar epithelial cells and type II pneumocytes in alveoli, particularly on proliferating and regenerating type II pneumocytes [4-6]. An official American Thoracic Society/European Respiratory Society statement proposed that a serum KL-6 level above $1000 \mathrm{U} / \mathrm{mL}$ at the initial examination in patients with stable-state ILD is associated with a worse prognosis [7-9]. In addition, a recent systematic review and metaanalysis reported that higher serum KL-6 levels were associated with an increased risk of $\mathrm{AE}$ of idiopathic pulmonary fibrosis (IPF) [10]. On the contrary, there are few reports of the clinical significance of the serum KL-6 level at the time of diagnosis of AEs, though we often see AEs of ILD patients with low KL-6 levels in the clinical setting $[11,12]$.

In the present retrospective study, clinical parameters were compared between high and low serum KL-6 patients at the time of diagnosis of AEs of ILDs to attempt to classify their clinical features according to the serum KL-6 level.

\section{Materials and Methods}

2.1. Study Location and Patients. The retrospective cohort study involved patients seen between 2014 and 2018 at Yokohama City University Hospital and Yokohama City University Medical Center. The medical data of 95 patients with acute or subacute IIPs, including AEs of nonspecific interstitial pneumonia and idiopathic pulmonary fibrosis (IPF), acute interstitial pneumonia, cryptogenic organizing pneumonia, drug-induced ILD, or AEs of CTD-ILDs treated with corticosteroid pulse therapy, were assessed. Patients who did not receive steroid pulse therapy or had sarcoidosis were excluded. Medical records at the time of diagnosis of AE were reviewed for data including age, sex, diagnosis of ILD, Charlson Comorbidity Index score (CCIS), blood parameters (partial pressure of oxygen in arterial blood/ fraction of inspired oxygen (P/F ratio)), KL-6 (normal: $<500 \mathrm{U} / \mathrm{mL}$ ), lactate dehydrogenase (LDH; normal: < $225 \mathrm{U} /$ L), surfactant protein-D (SP-D; normal: $<110 \mathrm{ng} / \mathrm{mL}$ ), highresolution CT (HRCT) findings, and treatment regimens, including sivelestat $\mathrm{Na}$ hydrate, anticoagulation therapy before steroid pulse therapy, steroid use before steroid pulse therapy, and macrolides [13]. The findings of HRCT were evaluated using the semiquantitative scoring method described by Ooi et al. [14]. Abnormalities on HRCT images of lungs were categorized as ground-glass opacity and honeycomb and scored based on the ratios (\%) of the disease in each of the six lung lobes (0\%: 0 points, $1-25 \%$ : 1 point, 26-50\%: 2 points, 51-75\%: 3 points, and 76\%: 4 points). Global scores were calculated by adding the scores for each abnormality in all lobes. Patients were classified as high serum KL-6 patients $(\geq 1000 \mathrm{U} / \mathrm{mL})$ and low serum KL-6 patients $(<1000 \mathrm{U} / \mathrm{mL})$, and the extracted data were compared between the two groups.

2.2. Diagnosis of ILDs. Subtypes of IIP were confirmed from physical, serological, HRCT, and lung pathological findings in accordance with the official statement for IIPs [7, 15]. Patients for whom lung biopsy could not be performed due to severe hypoxemia were diagnosed based on the HRCT classification $[7,15]$. The CTD-ILD diagnosis was confirmed by physical, serological, and HRCT findings consistent with ILD, and lung biopsy was undertaken to exclude other pulmonary diseases. A diagnosis of drug-induced ILD was based on previously reported criteria [16]. An AE of ILD was defined as worsening of hypoxemia reflecting severely impaired gas exchange; worsening of dyspnoea; newly appeared alveolar infiltration on radiography; and absence of alternative aetiologies including pneumothorax, pulmonary embolism, infection, or heart failure [7, 17-20].

2.3. Statistical Analysis. Data were statistically analysed using JMP 12 (SAS Institute Inc., Cary, NC, USA) and were shown as medians with 25 th-75th percentiles or numbers (\%). Groups were compared using the Wilcoxon rank-sum test or Pearson's chi-squared test. Optimal parameter cutoff values were determined from receiver operating characteristic (ROC) curves. Survival curves were generated using the Kaplan-Meier method and compared using log-rank tests. Predictors of 6-month mortality were determined using multiple stepwise regression analysis. Values with $P<0.05$ were considered significant.

2.4. Study Approval. This research was performed in accordance with the Declaration of Helsinki and approved by the institutional review board at Yokohama City University Hospital (approval no. B171100003). In this retrospective study, consent for participation was obtained by disclosing the clinical study with the description of the opt-out process (https://www.yokohama-cu.ac.jp/amedrc/ethics/ethical/f uzoku_optout.html). The severely ill condition or deep sedation of AE-ILD patients precluded us from obtaining informed consent from the patients themselves. Therefore, written informed consent was obtained from the patients' relatives or their legal guardians.

\section{Results}

3.1. Patient Characteristics. Table 1 shows the clinical characteristics of the patients with AEs of ILDs; there were 38 (40\%) patients with high serum KL-6 and 57 (60\%) patients with low serum KL-6 levels. The diagnoses of the 95 patients who were all treated with corticosteroid pulse therapy were AE of idiopathic ILDs in 62 patients (65\%) and AE of secondary ILDs in 33 patients (35\%). There was no significant difference in the diagnoses between the high and low serum KL-6 groups. Other clinical parameters including age, sex, CCIS, symptom onset, blood biomarkers (P/F ratio and SP-D), ground-glass opacity scores calculated from HRCT, and treatment regimens except serum $\mathrm{LDH}$ and honeycomb score showed similar tendencies between these groups. The main cause of death in the high and low serum KL-6 groups was AE, and there was no difference in the cause of death between these two groups. High serum KL-6 patients with AEs of idiopathic or secondary ILDs and low 
TABLe 1: Patients' characteristics.

\begin{tabular}{|c|c|c|c|c|}
\hline Characteristics & $\begin{array}{l}\text { High serum KL-6 } \\
\qquad(N=38)\end{array}$ & $\begin{array}{l}\text { Low serum KL-6 } \\
\quad(N=57)\end{array}$ & $\begin{array}{l}\text { Total patients } \\
\quad(N=95)\end{array}$ & $\begin{array}{l}P \text { values (high KL-6 vs. low } \\
\text { KL-6) }\end{array}$ \\
\hline Age, $y$ & $75(71-80)$ & $76(70-81)$ & $75(71-80)$ & 0.761 \\
\hline Male sex & $24(63)$ & $44(77)$ & $68(72)$ & 0.137 \\
\hline CCIS & $2(1-4)$ & $2(1-4)$ & $2(1-4)$ & 0.832 \\
\hline $\begin{array}{l}\text { From symptom onset to treatment, } \\
\text { days }\end{array}$ & $7(2.5-19)$ & $6(2.5-14)$ & $6(2.8-15)$ & 0.840 \\
\hline \multicolumn{5}{|l|}{ Diagnosis of $\mathrm{AE}$} \\
\hline IPF & $8(21)$ & $9(16)$ & $17(18)$ & 0.589 \\
\hline Others & $20(53)$ & $25(44)$ & $45(47)$ & 0.412 \\
\hline \multicolumn{5}{|l|}{ Secondary ILDs } \\
\hline CTD-ILD & $6(16)$ & $13(23)$ & $19(20)$ & 0.445 \\
\hline Drug & $3(8)$ & $10(17)$ & $13(14)$ & 0.232 \\
\hline Others & $1(2)$ & $0(0)$ & $1(1)$ & 0.400 \\
\hline \multicolumn{5}{|l|}{ Biomarkers } \\
\hline $\mathrm{P} / \mathrm{F}$ ratio & $274(218-308)$ & $248(152-311)$ & $268(187-309)$ & 0.329 \\
\hline $\mathrm{LDH}, \mathrm{IU} / \mathrm{L}$ & $321(268-446)$ & $277(216-375)$ & $282(235-405)$ & 0.013 \\
\hline SP-D, ng/mL & $337(137-541)$ & $207(140-364)$ & $233(138-409)$ & 0.112 \\
\hline \multicolumn{5}{|l|}{ HRCT scores } \\
\hline GGO scores & $10(6-13.8)$ & $10(8-15.5)$ & $10(7-15)$ & 0.729 \\
\hline Honeycomb & $3(0-7)$ & $0(0-4)$ & $1(0-5)$ & 0.045 \\
\hline \multicolumn{5}{|l|}{ Treatment } \\
\hline PSL before pulse & $11(29)$ & $10(18)$ & $21(22)$ & 0.189 \\
\hline PSL pulse & $38(100)$ & $57(100)$ & $95(100)$ & 1.000 \\
\hline PSL after pulse & $11(29)$ & $23(40)$ & $34(36)$ & 0.256 \\
\hline Macrolide & $8(21)$ & $12(21)$ & $20(21)$ & 1.000 \\
\hline NEI & $3(8)$ & $8(14)$ & $11(12)$ & 0.360 \\
\hline Anticoagulant & $6(16)$ & $12(21)$ & $18(19)$ & 0.521 \\
\hline \multicolumn{5}{|l|}{ Outcome } \\
\hline Six-month mortality & $12(32)$ & $15(26)$ & $27(28)$ & 0.685 \\
\hline \multicolumn{5}{|l|}{ Cause of mortality } \\
\hline $\mathrm{AE}$ & $11(29)$ & $14(25)$ & $25(26)$ & 0.634 \\
\hline Lung cancer & $1(3)$ & $1(2)$ & $2(2)$ & 0.771 \\
\hline
\end{tabular}

Results are shown as medians with 25th-75th percentiles or numbers (\%). Serum SP-D could be measured in 92 patients (97\%). AE: acute exacerbation; CCIS: Charlson Comorbidity Index score; CVD-IP: collagen vascular disease-related interstitial pneumonia; GGO: ground-glass opacity; HRCT: high-resolution computed tomography; ILD: interstitial lung disease; IPF: idiopathic pulmonary fibrosis; KL-6: Krebs von den Lungen-6; LDH: lactate dehydrogenase; NEI: neutrophil elastase inhibitor; P/F ratio: partial pressure of oxygen in arterial blood/fraction of the inspiratory oxygen; PSL: prednisolone; SP-D: surfactant protein-D.

serum KL-6 patients with AEs of idiopathic or secondary ILDs had similar 6-month mortality rates (Figure 1).

3.2. Stepwise Multiple Logistic Regression Analysis. In both patients with high and low serum KL-6 levels, clinical parameters including age, sex, CCIS, diagnosis of ILDs, $\mathrm{P} / \mathrm{F}$ ratio, serum $\mathrm{LDH}$ and $\mathrm{SP}-\mathrm{D}$, and the GGO and honeycomb scores were evaluated using stepwise multiple logistic regression analysis, whereas serum LDH was a significant predictor of 6-month mortality in high serum KL-6 patients (OR: 1.006; 95\% CI: 1.003-1.009; $P<0.001$ ), and CCIS (OR: 1.502; 95\% CI: 1.242-1.838; $P<0.001)$ and sex (OR: 5.751; 95\% CI: $1.121-105.163 ; P=0.033$ ) were significant predictors in low KL-6 patients (Table 2). In the patients with low serum KL-6 levels, the area under the ROC curve (AUC) was 0.541 in the evaluation of serum $\mathrm{LDH}$ as a predictor of 6 month mortality (Figure 2(a)). The 38 patients were assigned to groups with either low $\mathrm{LDH}(N=11)$ or high $\mathrm{LDH}$
$(N=46)$ levels based on the optimal cutoff $(206 \mathrm{IU} / \mathrm{mL})$. Log-rank tests showed that the Kaplan-Meier survival curves of these groups did not differ significantly $(P=0.227)$ (Figure 2(a)). On the contrary, in the patients with high serum KL-6 levels, the AUC was 0.897 in the evaluation of serum $\mathrm{LDH}$ as a predictor of 6-month mortality (Figure 2(b)). The optimal cutoff LDH level for estimating 6month mortality was $381 \mathrm{IU} / \mathrm{mL}(P<0.001)$. The 38 patients were assigned to groups with either low serum $\mathrm{LDH}(N=23)$ or high serum LDH $(N=15)$ levels based on this cutoff. Logrank tests showed that the Kaplan-Meier survival curves of these groups differed significantly $(P<0.001)$ (Figure $2(b)$ ).

3.3. Relationship between 6-Month Mortality and CCIS. In the patients with low serum KL-6 levels, the AUC was 0.836 in the evaluation of CCIS as a predictor of 6-month mortality (Figure 3(a)). The optimal cutoff CCIS value for predicting 6 -month mortality was 4 points $(P<0.001)$. The 


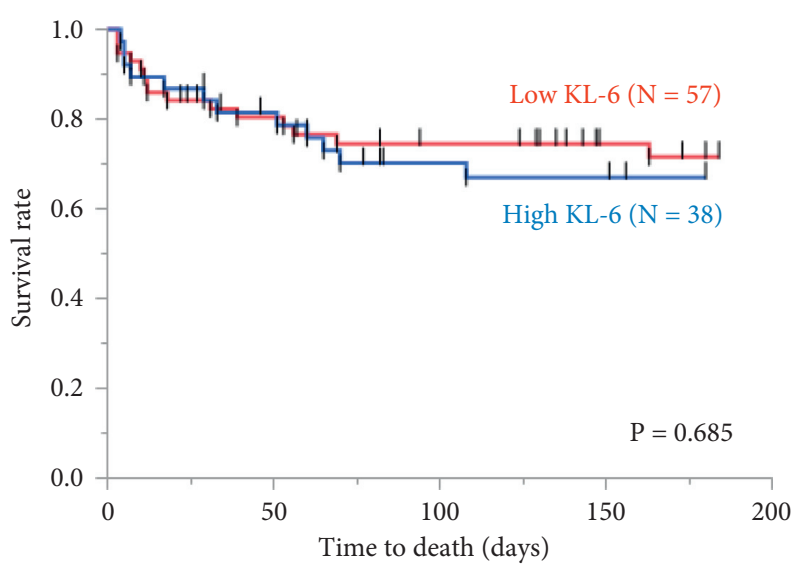

(a)

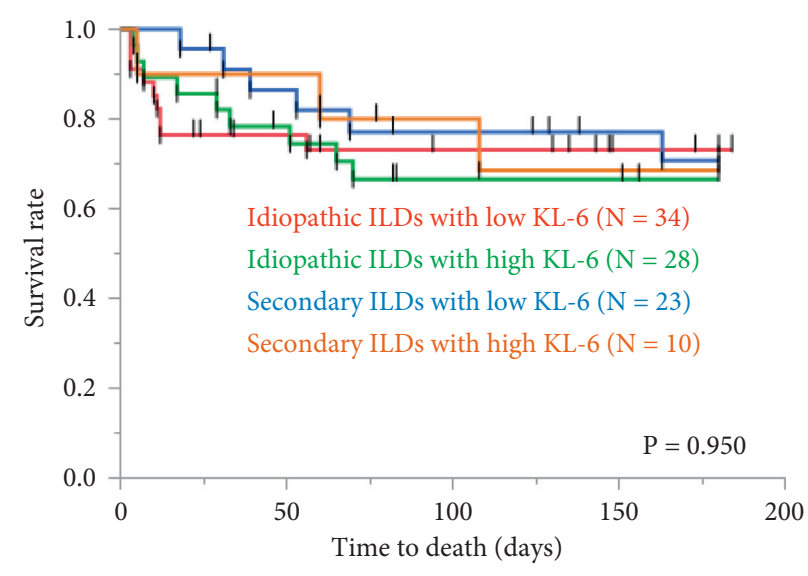

(b)

Figure 1: Comparison between high serum KL-6 and low serum KL-6 patients. The enrolled patients consist of 38 (40\%) patients with high serum KL-6 (idiopathic: 28 patients and secondary: 10 patients) and 57 (60\%) patients with low serum KL-6 (idiopathic: 34 patients and secondary 23 patients) levels. There is no significant difference in the 6-month prognosis between the high and low serum KL-6 patients $(P=0.685)(a)$. In addition, high serum KL-6 patients with AEs of idiopathic or secondary ILDs and low serum KL-6 patients with AEs of idiopathic or secondary ILDs have similar 6-month outcomes $(P=0.950)(b)$. ILD: interstitial lung disease; KL-6: Krebs von den Lungen-6.

TABLE 2: Multiple stepwise regression analysis of primary predictors of 6-month mortality (age, sex, CCIS, diagnosis, LDH, P/F ratio, ground-glass opacity, and honeycomb scores).

\begin{tabular}{lccc}
\hline Variable & $\begin{array}{c}95 \% \text { confidence } \\
\text { interval }\end{array}$ & Odds ratio & $\begin{array}{c}P \\
\text { values }\end{array}$ \\
\hline (A) Low KL-6 group & 1.502 & $1.242-1.838$ & $<0.001$ \\
CCIS & 5.751 & $1.121-105.163$ & 0.033 \\
Sex, male vs. & 1.002 & $1.000-1.005$ & 0.058 \\
female & & & \\
Serum LDH & 1.006 & $1.003-1.009$ & $<0.001$ \\
\hline (B) High KL-6 group &
\end{tabular}

CCIS: Charlson Comorbidity Index score; LDH: lactate dehydrogenase; P/F ratio: partial pressure of oxygen in arterial blood/fraction of inspired oxygen.

57 patients were assigned to groups with either low CCIS $(N=41)$ or high CCIS $(N=16)$ levels based on this cutoff value. Log-rank tests showed that the Kaplan-Meier survival curves of these groups differed significantly $(P<0.001)$ (Figure 3(a)). On the contrary, in the patients with high serum KL-6 levels, the AUC was 0.663 in the evaluation of CCIS as a predictor of 6-month mortality (Figure $3(\mathrm{~b})$ ). The 57 patients were assigned to groups with either low CCIS $(N=27)$ or high CCIS $(N=11)$ levels based on the same cutoff value. Log-rank tests showed that the Kaplan-Meier survival curves of these groups did not differ significantly $(P=0.083)$ (Figure 3(b)).

3.4. Incidence of Complications according to the Serum KL-6 Level and 6-Month Outcomes. Figure 4 shows a comparison of comorbidities in survivors with low serum KL-6 levels (A), nonsurvivors with low KL-6 levels (B), survivors with high serum KL-6 levels (C), and nonsurvivors with high serum KL-6 levels (D), respectively, from the left bar. The incidences of congestive heart failure $(12 \%, 33 \%, 0 \%$, and $17 \%)$, symptomatic chronic pulmonary disease $(29 \%, 73 \%$, $46 \%$, and $50 \%)$, cerebrovascular disease $(2 \%, 27 \%, 4 \%$, and $8 \%)$, and second metastatic solid tumours (2\%, 33\%, $4 \%$, and $17 \%)$ were the highest in nonsurvivors with low serum KL-6 levels (all $P<0.05)$.

\section{Discussion}

Serum KL-6 measurement is thought to be useful for detecting the presence of ILDs, evaluating ILD activity, and predicting the prognosis in various types of ILDs [21]. Several other clinical studies have proposed that serum KL-6 could predict the incidence of AEs, which are the most common cause of death in patients with ILD $[9,10,22]$. On the contrary, there are few reports of the relationship between the serum KL- 6 levels at the time of diagnosis of AE and these disease outcomes. Though it has been reported that serum $\mathrm{LDH}$ (cutoff value: $280 \mathrm{IU} / \mathrm{L}$ ), KL-6 (cutoff value: $1000 \mathrm{IU} / \mathrm{L}$ ), P/F ratio (cutoff value: 100), and extent of abnormal HRCT findings were significant predictors of 3-month mortality in IPF patients with an AE, we often saw patients with a poor prognosis despite a normal KL-6 level at the time of AE diagnosis $[11,12]$. Interestingly, in the present study, the ILD patients with high and low serum KL-6 levels had similar mortality, and it was shown that the prognostic factors were different between the two groups (high serum KL-6 group: serum LDH level; low serum KL-6 group: CCIS and sex).

A high KL-6 level was reported to be associated with the extent of lung fibrosis, which reflected regeneration of type II pneumocytes and/or enhancement of permeability following the destruction of the air-blood barrier in the affected lung [23-25]. An increased serum LDH level, which is a nonspecific biomarker, reflects lung inflammation and cellular 

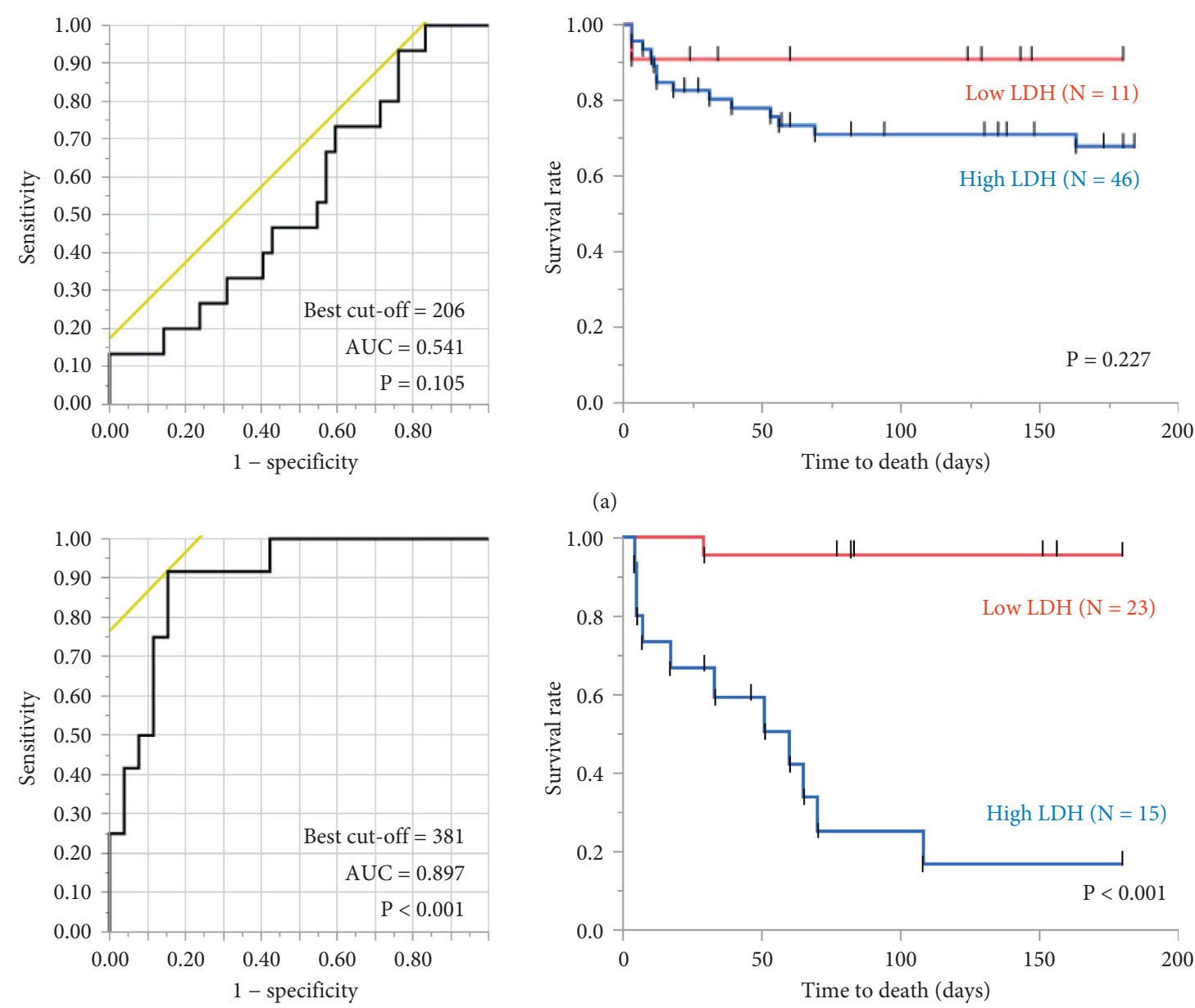

(a)

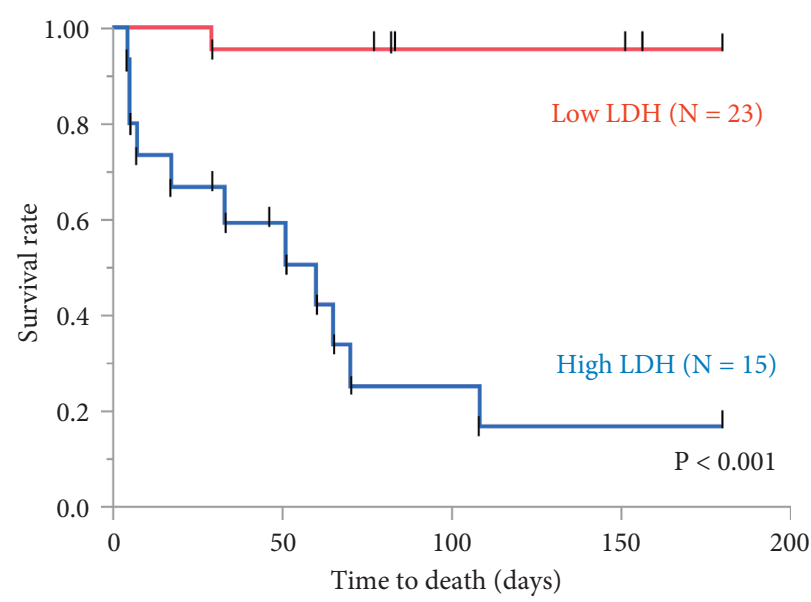

(b)

FIGURE 2: Relationship between 6-month mortality and serum LDH levels. In patients with low serum KL-6 levels, the AUC is 0.541 in the evaluation of serum LDH as a predictor of 6-month mortality (a). Log-rank tests show that the Kaplan-Meier survival curves of these groups do not differ significantly $(P=0.227)(\mathrm{A})$. On the contrary, in the patients with high serum KL-6 levels, the AUC value is 0.897 in the evaluation of serum LDH as a predictor of 6-month mortality (b). Log-rank tests show that the Kaplan-Meier survival curves of these groups differ significantly $(P<0.001)(B)$. AUC: area under the ROC curve; LDH: lactate dehydrogenase; KL-6: Krebs von den Lungen-6.

damage in patients with ILD [26-28]. The present study showed that high serum KL-6 patients at the AE diagnosis presented a greater extent of fibrosis of HRCT, higher serum LDH levels, and a significant increase of serum KL6 from stable condition than the low serum KL-6 patients (Supplementary Table). In addition, in the high serum KL6 group, patients with high serum LDH levels were found to have higher GGO scores calculated from HRCT (13 points vs. 9 points $(P<0.001))$ and lower $\mathrm{P} / \mathrm{F}$ ratios $(223$ vs. $296(P=0.004))$ than those with low serum LDH levels. From the above, patients with high serum KL-6 and LDH levels were considered to have more severe DAD with strong inflammation and increased permeability of the alveolar-capillary barrier and ongoing progressive fibrosis.

Serum KL-6 has been reported to be a significant prognostic factor in AEs of ILDs, but the serum KL-6 levels at the time of AE diagnosis are wide ranging [11]. In clinical practice, we also see patients whose serum KL-6 levels are not very high while meeting the diagnostic criteria for $\mathrm{AE}$ [12]. In the present study, there proved to be no difference in 6-month mortality between the high and low serum KL-6 patients. There are several possible reasons for this. First, comorbidities significantly affect the clinical course of ILD [29]. A retrospective cohort study of 272 patients with IPF suggested that there was a significant negative impact of arteriosclerosis, other cardiovascular diseases (mainly valvular heart disease, cardiac arrhythmias, and dilated cardiomyopathy), lung cancer, and pulmonary and cancer comorbidities on survival [29]. Another IPF cohort study that included 65 patients reported that baseline cardiovascular diseases were the predictors of an AE of IPF [30]. In the present study, the comparison of comorbidities between survivors with low serum KL-6, nonsurvivors with low KL-6, survivors with high serum KL-6, and nonsurvivors with high serum KL-6 levels showed that the incidences of congestive heart failure, symptomatic chronic pulmonary disease, cerebrovascular disease, and second metastatic solid tumours were significantly higher in nonsurvivors 

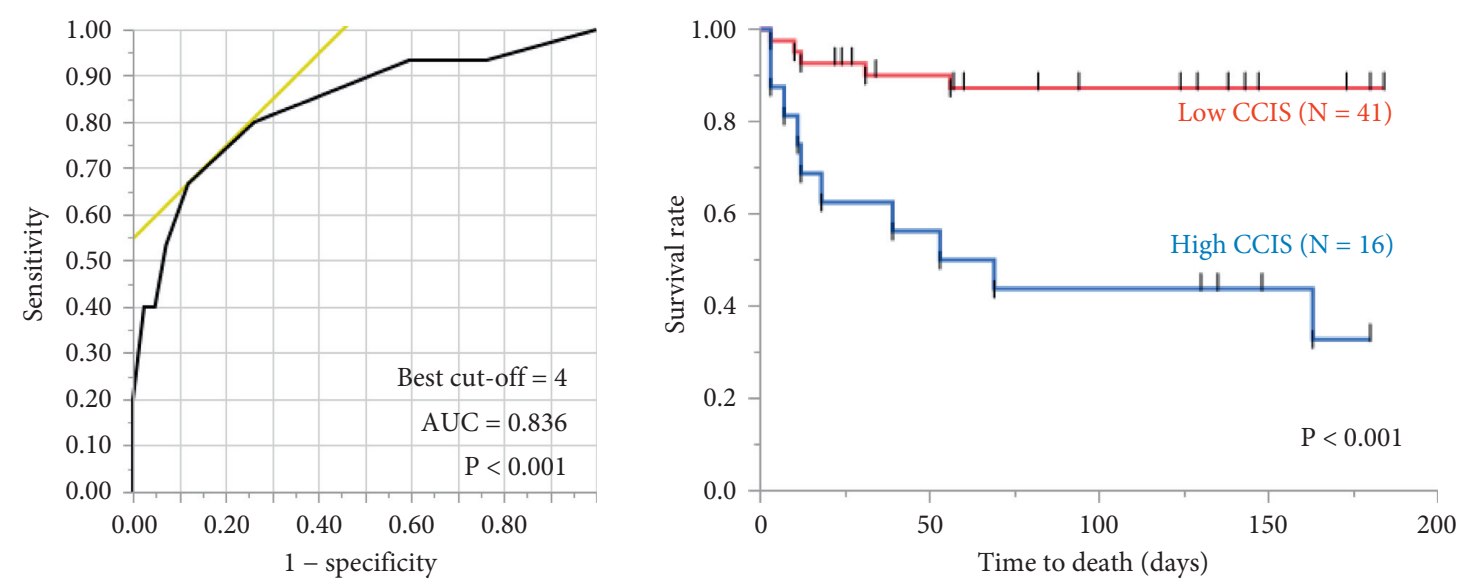

(a)
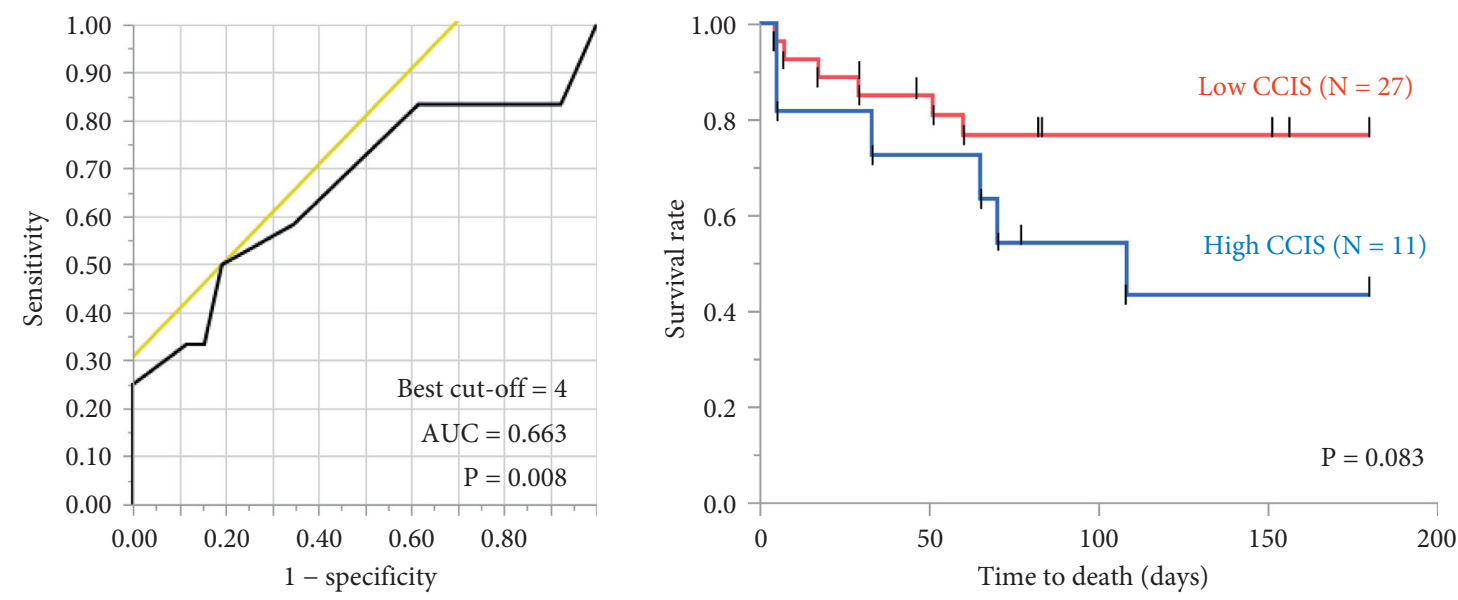

(b)

FIGURE 3: Relationship between 6-month mortality and CCIS. In the patients with low serum KL-6 levels, the AUC is 0.836 in the evaluation of CCIS as a predictor of 6-month mortality (a). The optimal cutoff CCIS for estimating 6-month mortality is 4 points $(P<0.001)$. Log-rank tests show that the Kaplan-Meier survival curves of these groups differ significantly $(P<0.001)(\mathrm{A})$. On the contrary, in the patients with high serum KL-6 levels, the AUC value is 0.663 in the evaluation of CCIS as a predictor of 6-month mortality (b). Log-rank tests show that the Kaplan-Meier survival curves of these groups do not differ significantly $(P=0.083)(B)$. AUC: area under the ROC curve; CCIS: Charlson Comorbidity Index score; KL-6: Krebs von den Lungen-6.

with low serum KL-6 levels than in the other groups. Second, the pathological findings in patients with AE-IPF represent not only DAD but also a variety of pathological conditions including OP, DAH, lung cancer, and bronchopneumonia [3]. In fact, comparing two autopsy cases enrolled in the present study, though HO-1, which is an oxidative stress marker, was expressed to the same extent in lung cells in both the high KL- 6 case and the low KL-6 case, in the former, DAD was the main component (Supplementary Figure S1, case 1 [31]), and in the latter, DAH and pulmonary vascular microthrombosis were the main components (DAD findings were minor) (Supplementary Figure S1, case 2 [12]). Consistent with these autopsy findings, our additional data showed that hemoglobin levels with the low serum KL-6 patients were significantly lower than those with the high serum KL-6 patients (Supplementary Table). These results suggest that patients with low serum KL-6 levels do not have severe
$\mathrm{DAD}$ and that various comorbidities and histological types such as DAH and vascular thrombosis may have a strong impact on prognosis.

The present study has some limitations. First, the study was limited by the small number of patients and the absence of additional validation datasets. In order to generalize these findings, further validation studies are essential. Second, the clinical diagnoses of the enrolled patients were heterogeneous, but there was no significant difference in the ILD diagnoses between the high and low serum KL-6 groups. Third, the low serum KL-6 group likely contained various pathological changes other than DAD, but pathological assessment was not performed after the onset of $\mathrm{AE}$ in all patients due to severe respiratory failure. Therefore, the credibility of this study will be increased by evaluating the relationship between clinical parameters such as blood examination and radiographic findings and prognosis in autopsy cases only. 


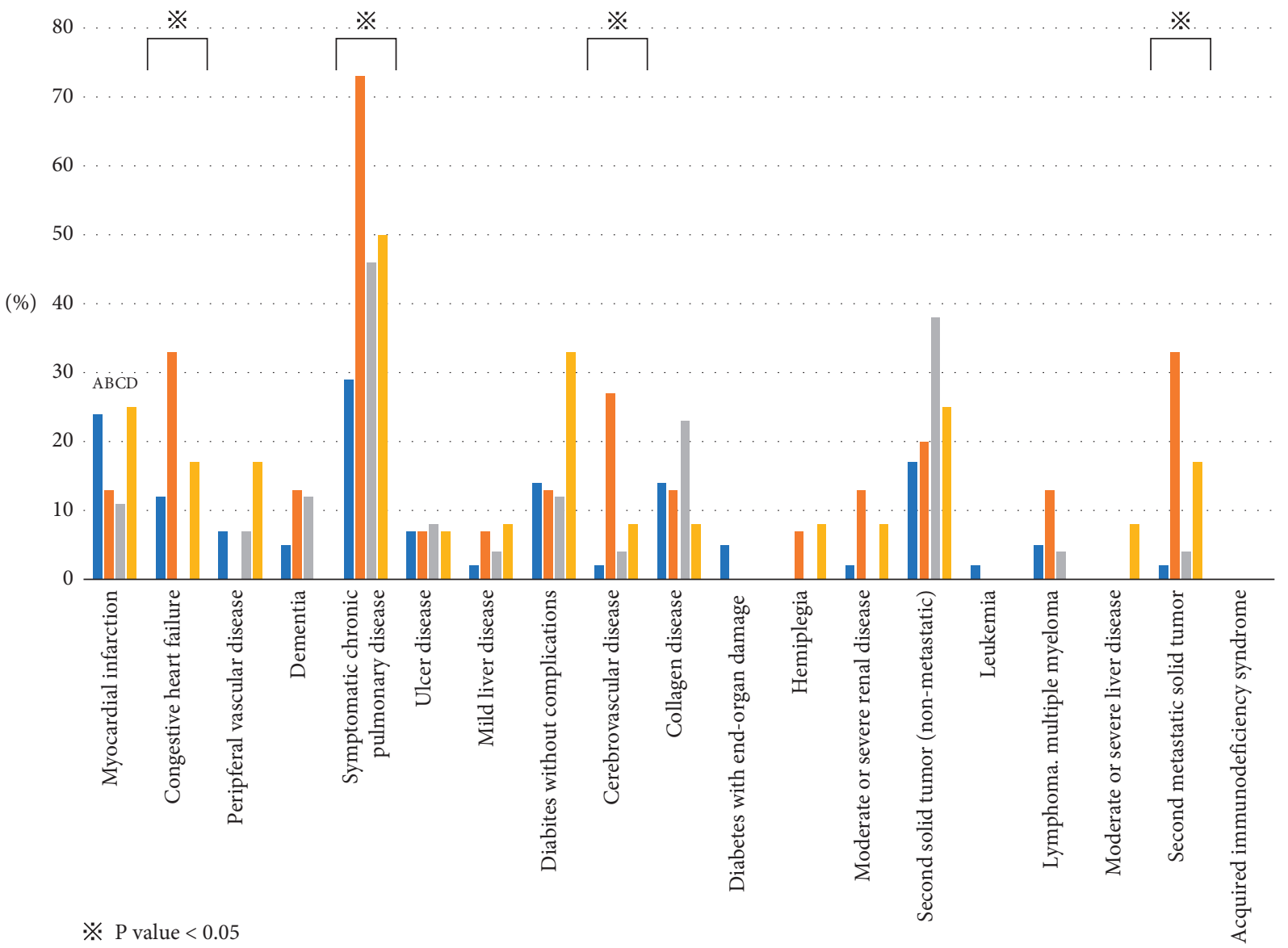

FIgURE 4: Incidence of complications according to serum KL-6 levels and 6-month outcomes. From the left bar, there are four groups, including survivors with low serum KL-6 (A), nonsurvivors with low serum KL-6 (B), survivors with high serum KL-6 (C), and nonsurvivors with high serum KL-6 (D) levels. The incidences of congestive heart failure, symptomatic chronic pulmonary disease, cerebrovascular disease, and second metastatic solid tumours are significantly the highest in nonsurvivors with low serum KL-6 levels (all $P<0.05$ ). KL-6: Krebs von den Lungen-6.

\section{Conclusions}

The clinical features of patients with AEs of ILDs may differ depending on the serum KL-6 level, and clinicopathological examination according to this subtyping guided by the serum KL-6 level is essential.

\section{Abbreviations}

AE: $\quad$ Acute exacerbation

AUC: Area under the ROC curve

CCIS: Charlson Comorbidity Index score

CI: $\quad$ Confidence interval

CTD- Connective tissue disease-associated ILD

ILD:

DAD: Diffuse alveolar damage

DAH: Diffuse alveolar haemorrhage

GGO: Ground-glass opacity

HRCT: High-resolution CT

HO-1: Heme oxygenase-1

IIPs: Idiopathic interstitial pneumonias

ILD: Interstitial lung disease

IPF: Idiopathic pulmonary fibrosis
KL-6: Krebs von den Lungen-6

LDH: Lactate dehydrogenase

NEI: Neutrophil elastase inhibitor

OP: Organizing pneumonia

OR: Odds ratio

P/F ratio: Partial pressure of oxygen in arterial blood/ fraction of inspired oxygen

PSL: $\quad$ Prednisolone

ROC: Receiver operating characteristic

SP-D: Surfactant protein-D.

\section{Data Availability}

The datasets used and/or analysed during the current study are available from the corresponding author upon reasonable request.

\section{Disclosure}

This manuscript has been presented as a preprint in Research Square according to the following link: https://www. researchsquare.com/article/rs-439754/v1. Also, the related 
article is available at https://www.researchgate.net/public ation/350830508_The_extent_of_honeycombing_on_comp uted_tomography_cannot_predict_the_treatment_outcome _of_patients_with_acute_exacerbations_of_interstitial_lun g_disease_Retrospective_cohort_study.

\section{Conflicts of Interest}

The authors declare that they have no conflicts of interest.

\section{Supplementary Materials}

Pathological findings at autopsy of AEs of ILDs [12, 31]. Figure legends: case 1 is a patient with high serum KL-6 (A: $\mathrm{HE}$ staining; C: immunohistochemical staining for $\mathrm{HO}-1$ expression) [31]. Case 2 is a patient with low serum KL-6 (B: $\mathrm{HE}$ staining; D: immunohistochemical staining for $\mathrm{HO}-1$ expression) [12]. (A) In addition to the fibrotic lesions that had passed for a relatively long time, oedematous thickening of the alveolar septum and vitreous membrane formation are observed focally. There is no evidence of granulomas or vasculitis. This is considered consistent with active DAD superimposed on fibrosis. (B) DAH and relatively mild DAD-like change are superimposed on fibrosis. DAH is the more predominant pattern than DAD. In addition, pulmonary vascular thrombosis is evident. This case is considered to be not typical for AE of ILD. (C, D) In both cases, high expression of HO-1 is similarly observed mainly in alveolar macrophages, whereas the expression of HO-1 in fibrotic lesions is not conspicuous. Abbreviations: AE: acute exacerbation; DAD: diffuse alveolar damage; DAH: diffuse alveolar haemorrhage; HE: haematoxylin-eosin staining; HO-1: heme oxygenase-1; KL-6: Krebs von den Lungen-6; ILD: interstitial lung disease. Supplementary Table: footnotes: $\Delta$ KL- 6 means the variation of serum KL-6 from a stable condition to acute exacerbation diagnosis. These data could be assessed in 52 patients (high KL-6 patients: 25 and low KL-6 patients: 27). D-dimer level could be measured in 78 patients (high KL-6 patients: 28 and low KL-6 patients: 50). Abbreviations: Hb: hemoglobin. (Supplementary Materials)

\section{References}

[1] M. Natsuizaka, H. Chiba, K. Kuronuma et al., "Epidemiologic survey of Japanese patients with idiopathic pulmonary fibrosis and investigation of ethnic differences," American Journal of Respiratory and Critical Care Medicine, vol. 190, no. 7, pp. 773-779, 2014.

[2] A. Suzuki, Y. Kondoh, K. K. Brown et al., "Acute exacerbations of fibrotic interstitial lung diseases," Respirology, vol. 25, no. 5, pp. 525-534, 2020.

[3] K. Oda, H. Ishimoto, S. Yamada et al., "Autopsy analyses in acute exacerbation of idiopathic pulmonary fibrosis," Respiratory Research, vol. 15, no. 1, p. 109, 2014.

[4] N. Kohno, S. Kyoizumi, Y. Awaya, H Fukuhara, M Yamakido, and M Akiyama, "New serum indicator of interstitial pneumonitis activity. Sialylated carbohydrate antigen KL-6," Chest, vol. 96, pp. 68-73, 1989.

[5] N. Kohno, Y. Awaya, T. Oyama et al., "KL-6, a mucin-like glycoprotein, in bronchoalveolar lavage fluid from patients with interstitial lung disease," American Review of Respiratory Disease, vol. 148, no. 3, pp. 637-642, 1993.

[6] S. Ohshimo, A. Yokoyama, N. Hattori, N. Ishikawa, Y. Hirasawa, and N. Kohno, "KL-6, a human MUC1 mucin, promotes proliferation and survival of lung fibroblasts," Biochemical and Biophysical Research Communications, vol. 338, no. 4, pp. 1845-1852, 2005.

[7] W. D. Travis, U. Costabel, D. M. Hansell et al., "An official American thoracic society/european respiratory society statement: update of the international multidisciplinary classification of the idiopathic interstitial pneumonias," American Journal of Respiratory and Critical Care Medicine, vol. 188, no. 6, pp. 733-748, 2013.

[8] A. Yokoyama, K. Kondo, M. Nakajima et al., "Prognostic value of circulating KL-6 in idiopathic pulmonary fibrosis," Respirology, vol. 11, no. 2, pp. 164-168, 2006.

[9] H. Satoh, K. Kurishima, H. Ishikawa, and M. Ohtsuka, "Increased levels of KL-6 and subsequent mortality in patients with interstitial lung diseases," Journal of Internal Medicine, vol. 260, no. 5, pp. 429-434, 2006.

[10] M. Qiu, Y. Chen, and Q. Ye, "Risk factors for acute exacerbation of idiopathic pulmonary fibrosis: a systematic review and meta-analysis," The Clinical Respiratory Journal, vol. 12, no. 3, pp. 1084-1092, 2018.

[11] T. Kishaba, H. Tamaki, Y. Shimaoka, H. Fukuyama, and S. Yamashiro, "Staging of acute exacerbation in patients with idiopathic pulmonary fibrosis," Lung, vol. 192, no. 1, pp. 141-149, 2014.

[12] K. Murohashi, Y. Hara, A. Aoki et al., "Diffuse alveolar hemorrhage complicating acute exacerbation of IPF," Respiratory Medicine Case Reports, vol. 29, Article ID 101022, 2020.

[13] M. E. Charlson, P. Pompei, K. L. Ales, and C. R. MacKenzie, "A new method of classifying prognostic comorbidity in longitudinal studies: development and validation," Journal of Chronic Diseases, vol. 40, no. 5, pp. 373-383, 1987.

[14] G. C. Ooi, M. Y. Mok, K. W. T. Tsang et al., "Interstitial lung disease in systemic sclerosis: an HRCT-clinical correlative study," Acta Radiologica, vol. 44, no. 3, pp. 258-264, 2003.

[15] G. Raghu, B. Rochwerg, Y. Zhang et al., "An official ATS/ERS/ JRS/ALAT clinical practice guideline: treatment of idiopathic pulmonary fibrosis. An update of the 2011 clinical practice guideline," American Journal of Respiratory and Critical Care Medicine, vol. 192, no. 2, pp. e3-e19, 2015.

[16] K. Kubo, A. Azuma, M. Kanazawa et al., "Consensus statement for the diagnosis and treatment of drug-induced lung injuries," Respiratory Investigation, vol. 51, no. 4, pp. 260-277, 2013.

[17] H. Taniguchi and Y. Kondoh, "Acute and subacute idiopathic interstitial pneumonias," Respirology, vol. 21, no. 5, pp. 810-820, 2016.

[18] H. R. Collard, C. J. Ryerson, T. J. Corte et al., "Acute exacerbation of idiopathic pulmonary fibrosis. An international working group report," American Journal of Respiratory and Critical Care Medicine, vol. 194, no. 3, pp. 265-275, 2016.

[19] I.-N. Park, D. S. Kim, T. S. Shim et al., "Acute exacerbation of interstitial pneumonia other than idiopathic pulmonary fibrosis," Chest, vol. 132, no. 1, pp. 214-220, 2007.

[20] R. Tachikawa, K. Tomii, H. Ueda et al., "Clinical features and outcome of acute exacerbation of interstitial pneumonia: collagen vascular diseases-related versus idiopathic," Respiration, vol. 83, no. 1, pp. 20-27, 2012.

[21] N. Ishikawa, N. Hattori, A. Yokoyama, and N. Kohno, "Utility of KL-6/MUC1 in the clinical management of interstitial lung 
diseases," Respiratory Investigation, vol. 50, no. 1, pp. 3-13, 2012.

[22] S. Ohshimo, N. Ishikawa, Y. Horimasu et al., "Baseline KL-6 predicts increased risk for acute exacerbation of idiopathic pulmonary fibrosis," Respiratory Medicine, vol. 108, no. 7, pp. 1031-1039, 2014.

[23] F. Okada, Y. Ando, K. Honda, S. Tanoue, S. Matsumoto, and H. Mori, "Comparison of pulmonary CT findings and serum KL-6 levels in patients with cryptogenic organizing pneumonia," British Journal of Radiology, vol. 82, no. 975, pp. 212-218, 2009.

[24] K. Sakamoto, H. Taniguchi, Y. Kondoh et al., "Serum KL-6 in fibrotic NSIP: correlations with physiologic and radiologic parameters," Respiratory Medicine, vol. 104, no. 1, pp. 127-133, 2010.

[25] Y. Hirasawa, N. Kohno, A. Yokoyama, Y. Inoue, M. Abe, and K. Hiwada, "KL-6, a human MUC1 mucin, is chemotactic for human fibroblasts," American Journal of Respiratory Cell and Molecular Biology, vol. 17, no. 4, pp. 501-507, 1997.

[26] M. Drent, N. A. M. Cobben, R. F. Henderson, E. F. M. Wouters, and M. van Dieijen-Visser, "Usefulness of lactate dehydrogenase and its isoenzymes as indicators of lung damage or inflammation," European Respiratory Journal, vol. 9, no. 8, pp. 1736-1742, 1996.

[27] R. A. DeRemee, "Serum lactic dehydrogenase activity and diffuse interstitial pneumonitis," Journal of the American Medical Association: The Journal of the American Medical Association, vol. 204, no. 13, pp. 1193-1195, 1968.

[28] S. Kanoh, H. Kobayashi, and K. Motoyoshi, "Exhaled ethane," Chest, vol. 128, no. 4, pp. 2387-2392, 2005.

[29] M. Kreuter, S. Ehlers-Tenenbaum, K. Palmowski et al., "Impact of comorbidities on mortality in patients with idiopathic pulmonary fibrosis," PLoS One, vol. 11, no. 3, Article ID e0151425, 2016.

[30] T. Kakugawa, N. Sakamoto, S. Sato et al., "Risk factors for an acute exacerbation of idiopathic pulmonary fibrosis," Respiratory Research, vol. 17, no. 1, p. 79, 2016.

[31] K. Harita, Y. Hara, and K. Murohashi, "A case of drug-induced lung injury induced by abiraterone which serum HO-1 levels reflected the disease activity," AJRS, vol. 9, pp. 141-146, 2020 . 